\title{
Injectable Antihyperglycemics: A Systematic Review and Critical Analysis of the Literature on Adherence, Persistence, and Health Outcomes
}

\author{
Carol M. Hamersky · Moshe Fridman · Cory L. Gamble • \\ Neeraj N. Iyer
}

Received: March 12, 2019 / Published online: May 3, 2019

(c) The Author(s) 2019

\section{ABSTRACT}

Introduction: Improving real-world medication adherence to injectable antihyperglycemics in type 2 diabetes mellitus (T2DM) is a clinical challenge. Quantification of the level of adherence required to achieve a minimal clinically important difference (MCID) in glycemic control would assist in meeting this goal. The study objective was to review the literature regarding the relationships of medication adherence and persistence with health outcomes in adult T2DM patients using injectable antihyperglycemics.

Methods: Systematic searches were conducted using electronic databases to identify publications over the last decade. Publications were

Carol M. Hamersky and Moshe Fridman contributed equally to this manuscript.

Enhanced Digital Features To view enhanced digital features for this article go to https://doi.org/10.6084/ m9.figshare.7976570.

Electronic Supplementary Material The online version of this article (https://doi.org/10.1007/s13300019-0617-3) contains supplementary material, which is available to authorized users.

C. M. Hamersky $(\bowtie)$ · C. L. Gamble · N. N. Iyer Novo Nordisk Inc., Plainsboro Township, NJ, USA e-mail: cahy@novonordisk.com

M. Fridman

AMF Consulting, Los Angeles, CA, USA screened against established eligibility criteria. Study data were extracted, evaluated, and used to identify strengths, limitations, and gaps in current evidence.

Results: Eligibility criteria were met by 38 studies, and this report analyzed 34 studies related to glycemic control $(n=25)$, healthcare resource use $(n=9)$, and healthcare costs $(n=14)$. Eight of these studies examined adherence to glucagon-like peptide- 1 receptor agonists (GLP-1 RA), including 1 study regarding adherence to GLP-1 RA or to insulin, and 1 study investigating a GLP-1 RA/insulin combination; the remaining studies involved insulin. Studies used a broad range of measures to classify adherence and persistence, and most measures were unable to reliably evaluate the complexities of patient behavior over time. Better adherence to injectable antihyperglycemic medications was generally found to be associated with improved glycemic control, although no studies attempted to identify a MCID. Although higher diabetes-related pharmacy and total healthcare costs were reported for adherent or persistent patients, these patients tended to have lower diabetes-related and all-cause medical costs.

Conclusion: Results of this review confirmed the effectiveness of injectable antihyperglycemic medications for glycemic control, suggesting that there are clinical and financial consequences to nonadherence. Although attempts were made to quantify the effects of 
nonadherence, the interpretation of study results was limited by the lack of a MCID and inadequate study design.

Funding: Novo Nordisk, Inc., Plainsboro Township, NJ, USA.

Plain Language Summary: Plain language summary available for this article.

Keywords: Glucagon-like peptide-1 receptor agonist; Insulin; Medication adherence; Medication compliance; Medication persistence; Real-world data; Type 2 diabetes

\section{PLAIN LANGUAGE SUMMARY}

From clinical trials, we know that antidiabetic medications are effective at improving health outcomes in adults with type 2 diabetes mellitus (T2DM). However, improving real-world patient adherence to injectable medications remains a clinical challenge. We performed a systematic review of the medical literature regarding realworld injectable medication adherence and health outcomes in adults with T2DM from 2007 onward using electronic databases. Eligibility criteria for the review were met by 38 studies, and this report analyzed 34 studies of the outcomes of glycemic control $(n=25)$, healthcare resource use $(n=9)$, and healthcare costs $(n=14)$. Eight studies examined adherence to glucagon-like peptide- 1 receptor agonists (GLP-1 RA), and the remaining studies involved insulin. Studies used a broad range of measures to classify adherence and persistence, and most measures were unable to reliably evaluate the complexities of patient behavior over time. Better adherence to injectable medications was generally found to be associated with improved glycemic control. Although higher diabetes-related pharmacy and total healthcare costs were reported for adherent or persistent patients, these patients had lower diabetes-related and all-cause medical costs. The results of this review confirmed the effectiveness of injectable medications for glycemic control, suggesting that there are clinical and financial consequences to nonadherence. However, the few studies yielded by this review were limited by inadequate study designs and did not produce evidence describing the level of adherence necessary to achieve glycemic control or other health outcomes. Future research in these areas would assist in the development of successful strategies for adherence improvement.

\section{INTRODUCTION}

The efficacy of antihyperglycemic medications for the improvement of glycemic control in patients with type 2 diabetes mellitus (T2DM) has been well established by clinical trial data [1]. In a recent review [2], Edelman and Polonsky argue that poor medication adherence is the key variable responsible for the gap between the clinical results achieved in randomized controlled clinical trials (RCT) and real-world studies. Theoretically, real-world studies should generally confirm the benefits of these medications with respect to improving clinical outcomes, and specifically confirm improvements in glycemic control. Here, in an attempt to quantify the consequences of nonadherence and identify strengths and limitations of this literature, we performed a systematic literature review regarding the effects of patient adherence to and persistence with T2DM injectable medications on health outcomes. The population of interest was adults with T2DM who used injectable antihyperglycemic medications (glucagon-like peptide-1 receptor antagonists [GLP-1 RA] and insulins).

\section{METHODS}

This systematic review conformed to Preferred Reporting Items for Systematic Reviews and Meta-Analyses (PRISMA) guidelines [3]. This article is based on previously conducted studies and does not contain any studies with human participants or animals performed by any of the authors. Search queries were performed for English language literature from January 1, 2007 through October 11, 2017 utilizing the Embase, PubMed, CINAHL, and Cochrane databases. This period covered the last decade of electronically available indexed studies from 
this study start date. Searches included adherence and persistence terms combined with terms to identify the T2DM population, and any injectable antihyperglycemic agent. See Appendix S1 in the Electronic supplementary material (ESM) for details.

Citations identified through the searches were assessed by one reviewer and verified by another based on the title and abstract, using predefined eligibility criteria (round 1). The inclusion criteria were: (1) study of a T2DM population; (2) study regarding outcomes of medication adherence or persistence; (3) analysis of injectable anthihyperglycemic medications; (4) inclusion of health outcomes data; and (5) allowable study types (observational studies and database/claims analyses). The exclusion criteria were: (1) oral treatments, infusions, on-demand treatments or treatments without a planned regimen, and treatments not self-injected by the patient; (2) studies with no data, e.g., protocol only studies; (3) case studies; (4) basic science studies; (5) meeting abstracts, dissertations, and pilot studies; (6) practice guidelines; (7) study population: animals; (8) study population: non-adults; (9) study population: patients with cancer; (10) study population: patients with gestational diabetes; and (11) reviews, commentaries, letters, or editorials.

For round 2, full text publications of potentially relevant citations were examined by two reviewers to assure that medication adherence was studied in relationship to the health outcome. For round 3, a final eligibility review for the remaining articles was conducted, and known studies not captured by this process were added to the selected publication list. Information was then extracted into a pre-defined database. Reviewer disagreements about study selection in the full text review and extraction phases were resolved by jointly re-examining studies and reaching mutual agreement.

Publications included in the review were assessed for risk of bias with the Newcastle-Ottawa Scale (NOS) [4]. Two reviewers assessed each article for NOS criteria. Any discrepancy in scoring was resolved by joint re-examination to arrive at consensus. NOS scores were subdivided into those indicating high quality $(\geq 6)$, moderate quality (4-5), and low quality (1-3).

\section{RESULTS}

\section{Study Selection and Characteristics}

Results of the literature search are described in Fig. 1. A total of 3491 publications were identified by the original searches, and 38 met all inclusion and exclusion criteria after a thorough review of the full text [5-42]. No studies from the search of the Cochrane database were eligible for the study, and this information was omitted from Fig. 1 for clarity. Although additional outcomes were identified in the 38 articles yielded by this review, this review focuses on studies of three health outcomes: (1) glycemic control, (2) healthcare resource use, and (3) healthcare costs. The other outcomes identified that are not reported here were: mortality ( 2 articles), weight change (4 articles), depression ( 1 article), health-related quality of life (4 articles), device satisfaction (1 article), hypoglycemic events (9 articles), and assorted clinical outcomes ( 1 article). This focus on the three outcomes of glycemic control, healthcare resource use, and healthcare costs limited the final selection to 34 studies, which excludes analysis of four of the studies retrieved [39-42]. Table 1 summarizes selected study characteristics. Quality scoring is presented in Table 2. Out of 9 potential total points per study, the median score was 5 (range 2-8).

To the extent possible, studies of GLP-1 RA and insulins are described separately, recognizing that these classes of medications have different adherence issues, e.g., GLP-1 RA is administered with an injection pen and should not produce wastage, in contrast to the use of both pens and syringes for insulins [43]. Also, insulin regimens may be more complex than GLP-1 RA regimens, a feature that has been associated with poorer adherence [44].

Studies regarding medication adherence typically distinguish between adherence (compliance) and persistence (non-discontinuation) [45]. Regarding adherence, see Table 1 for a listing of the various adherence measures used 


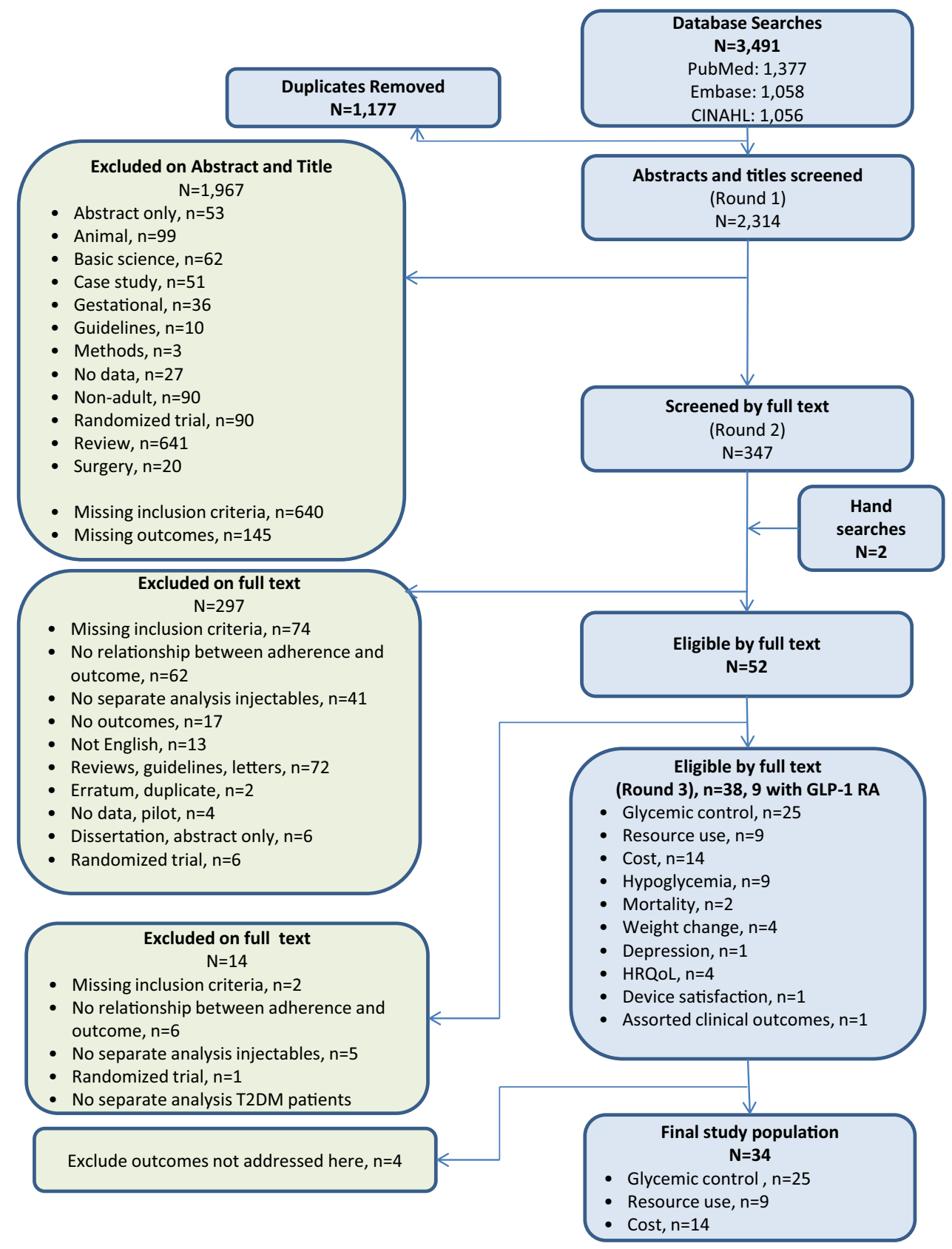

Fig. 1 PRISMA flow chart. Text in green boxes describes exclusions

in the final dataset. The main persistence measure was discontinuation at or before 1 year from index date. Although, in theory, discontinuation can be thought of as an extreme case of nonadherence, these concepts measure different types of departures from a treatment regimen and have different practical challenges in measurement. For administrative/claims data studies, It was common to use proportion of days covered (PDC) or medication possession ratio (MPR) $\geq 80 \%$ as a definition of adherence, and a gap of $\leq 90$ days was defined as being persistent. None of the reviewed studies considered primary nonadherence, i.e., patients who had not filled a prescription even once. With two exceptions, information regarding the 
Table 1 Summary of included studies

\section{Number of included studies}

A total of 34 publications that reported on the relationships between adherence to injectable antihyperglycemic medications and health outcomes in patients with T2DM were included

All studies but two identified relied on unique study populations; otherwise, there was no overlap between these studies. Two studies used the same dataset (Linetsky et al. [10] and Linetsky et al. [22]), evaluating data from the same source and same study population but using different study designs

\section{Study design}

In total, there were 17 studies based on commercial administrative/claims data $[5,7,8,14,15,19,25-35], 1$ based on US veterans' data (linked claims and medical records) [11], 12 studies based on medical records $[10,12,16,17,20-24,36-38]$ (6 of these also used surveys $[10,12,21-24]$ and 1 used Geisinger electronic medical records [17]), 2 based on national surveys $[9,13], 1$ based on a physician survey [6], and 1 based on a diabetic registry with survey [18]

Of the 34 included studies, 24 were retrospective cohort studies, 3 were prospective cohort studies $[21,24,38]$, and 7 were cross-sectional studies $[6,9,10,12,13,22,23]$

\section{Study population}

8 studies reported on GLP-1 RA with or without insulins [31-38], and 26 studies reported exclusively on insulins

20 studies involved the initiation of an injectable or a switch to a new injectable or device (8 involved GLP-1 RA), and 14 studies were focused on populations that were continuing with injectables (all involved insulin)

$[6,10-13,16-18,20,22-24,27,28]$

\section{Adherence measures}

Across the 34 included studies

2 used the Morisky Medication Adherence Scale $[13,24]$

1 used the Morisky Insulin Adherence Scale [12]
Table 1 continued

Other types of surveys used for adherence:

1: identified continuers, interrupters, and discontinuers [9]

1: identified differences in insulin timing [23]

1: identified dosing irregularities [6]

4: missed shots [10, 18, 21, 22]

1 used adequate refills for an entire quarter [15]

2 used the medication possession ratio

$(\mathrm{MPR}) \geq 80 \%[11,26]$

1 used the MPR in 5 categories [28]

1 used the adjusted MPR $\geq 80 \%$ [27]

3 used proportion of days covered $\geq 80 \%$

$[16,20,33]$

2 used proportion of days covered $\geq 80 \%$ and persistence at 1 year $[34,35]$

9 reported on persistence at 1 year

$[5,7,8,14,17,19,25,31,36]$

2 reported on persistence at 2 years [29, 32]

1 reported on persistence at 2 years + assorted clinical outcomes [30]

1 reported on persistence at 132 weeks [38]

1 reported on persistence at 6 months [37]

All identified methods to measure adherence were indirect, with no studies identified that measured adherence directly through observation of medication-taking or biological fluid samples

\section{Effects of adherence on disease outcomes}

The 34 studies reported on the following outcomes: glycemic control $(N=25)$

$[5,6,9-13,15-24,31-38]$, resource use $(N=9)[5,7,12,13,15,17,25,29,31]$, and costs $(N=14)[1,8,9,14,15,17,25-31,34]$

T2DM type 2 diabetes mellitus, GLP-1 RA glucagonlike peptide-1 receptor agonist, $M P R$ medication possession ratio 
Table 2 Quality scoring: quality assessment of included publications using the Newcastle-Ottawa Scale

\begin{tabular}{|c|c|c|c|c|c|c|}
\hline References & $\begin{array}{l}\text { Last name of first } \\
\text { author }\end{array}$ & Year & $\begin{array}{l}\text { Selection } \\
(\text { maximum 4) })^{* * * *}\end{array}$ & $\begin{array}{l}\text { Comparability } \\
(\text { maximum 2) }\end{array}$ & $\begin{array}{l}\text { Outcome } \\
(\text { maximum 3) }\end{array}$ & $\begin{array}{l}\text { Total } \\
\text { score }\end{array}$ \\
\hline [11] & Egede & 2014 & *** & ** & *** & 8 \\
\hline$[16]$ & Donnelly & 2007 & $* * *$ & $* *$ & ${ }^{* * *}$ & 8 \\
\hline [27] & Kleinman & 2008 & $* * *$ & ** & $* * *$ & 8 \\
\hline$[5]$ & Wei & 2014 & $* * *$ & $* *$ & $* *$ & 7 \\
\hline [14] & Anderten & 2015 & $* *$ & $* *$ & $* * *$ & 7 \\
\hline [15] & Ayyagari & 2015 & $* * *$ & $*$ & $* * *$ & 7 \\
\hline [26] & Cobden & 2007 & $* * *$ & $* *$ & $* *$ & 7 \\
\hline [29] & Perez-Nieves & 2016 & $* * *$ & $* *$ & $* *$ & 7 \\
\hline [30] & Kalirai & 2017 & $* * *$ & $* *$ & $* *$ & 7 \\
\hline [31] & Lin & 2017 & $* * *$ & $* *$ & $* *$ & 7 \\
\hline [33] & Durden & 2016 & $* * *$ & ${ }^{* *}$ & ** & 7 \\
\hline [34] & Buysman & 2015 & $* * *$ & ${ }^{* *}$ & ** & 7 \\
\hline [8] & Ascher-Svanum & 2014 & ${ }^{* * *}$ & ${ }^{* *}$ & * & 6 \\
\hline [17] & $\mathrm{Wu}$ & 2012 & $* * *$ & * & ** & 6 \\
\hline [19] & Sambamoorthi & 2017 & $* * *$ & $* *$ & $*$ & 6 \\
\hline [35] & Carls & 2017 & ** & ** & ** & 6 \\
\hline [10] & Linetsky & 2017 & $* *$ & $* *$ & $*$ & 5 \\
\hline$[12]$ & Osborn & 2016 & $* *$ & $* *$ & * & 5 \\
\hline [22] & Linetzky & 2016 & $* *$ & $* *$ & * & 5 \\
\hline [24] & Aikens & 2013 & $* *$ & $*$ & $* *$ & 5 \\
\hline [28] & Chandran & 2015 & $* * *$ & & $* *$ & 5 \\
\hline [32] & Levin & 2014 & $* * *$ & & $* *$ & 5 \\
\hline [36] & Buysschaert & 2010 & $* * *$ & & $* *$ & 5 \\
\hline$[7]$ & Wang & 2013 & $* * *$ & & * & 4 \\
\hline$[18]$ & Mashitani & 2013 & $* *$ & $*$ & $*$ & 4 \\
\hline [20] & Kindmalm & 2007 & $* *$ & $*$ & * & 4 \\
\hline [21] & Yavuz & 2015 & $* *$ & $*$ & $*$ & 4 \\
\hline$[25]$ & Hadjiyianni & 2017 & ** & * & * & 4 \\
\hline$[38]$ & Ivanyi & 2012 & $*$ & & $* * *$ & 4 \\
\hline [13] & DiBonaventura & 2014 & * & $* *$ & & 3 \\
\hline [23] & Nishimura & 2017 & * & $*$ & $*$ & 3 \\
\hline [37] & Varanasi & 2011 & $*$ & & $* *$ & 3 \\
\hline
\end{tabular}


Table 2 continued

\begin{tabular}{|c|c|c|c|c|c|c|}
\hline References & $\begin{array}{l}\text { Last name of first } \\
\text { author }\end{array}$ & Year & $\begin{array}{l}\text { Selection } \\
(\text { maximum 4) }\end{array}$ & $\begin{array}{l}\text { Comparability } \\
(\text { maximum } 2)^{* *}\end{array}$ & $\begin{array}{l}\text { Outcome } \\
(\text { maximum 3)*** }\end{array}$ & $\begin{array}{l}\text { Total } \\
\text { score }\end{array}$ \\
\hline$[6]$ & Leiter & 2015 & $*$ & & * & 2 \\
\hline [9] & Perez-Nieves & 2017 & $* *$ & & & 2 \\
\hline
\end{tabular}

type of provider prescribing the medication was not included in the published studies.

\section{GLYCEMIC CONTROL RESULTS}

\section{Studies Regarding the Impact of Medication Adherence and Persistence on Glycemic Control}

A total of 25 studies reported relationships between medication adherence or persistence and glycemic control [5, 6, 9-13, 15-24, 31-38]. Of these, 8 reported regarding GLP-1 RA use [31-38]. Data collection for all GLP-1 RA studies pertained to the use of exenatide or liraglutide, or, if not specifically stated, occurred before weekly formulations were approved by the United States Food and Drug Administration (2012) [46]. One of these studies [32] reported results for both medication types, and 1 study [31] reported results for a combination of GLP-1 RA and insulin. The remaining 26 studies reported exclusively on insulins.

\section{Improvements in Glycemic Control}

Most studies found that adherence or persistence had a positive effect on glycemic control, i.e., increased adherence or persistence was associated with decreased HbA1c, which was the predominant measure of glycemic control. Study designs varied widely, precluding any rigorous uniform summation. See Table 3 for individual study details.

\section{GLP-1 RA Studies}

Of the 8 GLP-1 RA articles, 5 reported improved HbA1c from baseline with persistence [31-38], although this was not statistically significant in 1 study [32] and not reported in another [36]. Four of these studies involved patients initiating GLP-1 RA and 1 involved initiation of a second class of medications, either GLP-1 RA or insulin, resulting in combination GLP-1 RA/insulin therapy [31]. Estimates of persistence with GLP-1 RA in these studies ranged from 17 to $86 \%$. As noted in Table 3, 1 study examined change in HbA1c at baseline for persisters only, 3 studies tested this change for persisters and nonpersisters, and 1 study presented an odds ratio for persisters vs nonpersisters regarding whether a HbA1c goal was met. Each study had a different design and study population, and follow-up periods ranged from 6 to 132 weeks.

The remaining 3 GLP-1 RA studies found improvements in $\mathrm{HbA1c}$ from baseline with adherence [33-35]. All 3 studies were for GLP-1 RA initiators, with varying combinations of prior and continuing medications, and were retrospective cohort studies using administrative/claims data. One of these studies examined both adherence and persistence in the same study population [34]. All 3 studies found a reduction in $\mathrm{HbA1}$ c with adherence defined as a PDC $\geq 80 \%$ ( $P$ values not reported for 1 study [35]), and 2 studies provided odds ratios for adherent vs nonadherent patients meeting HbA1c goals [33, 34]. Although these adherence studies used similar data sources and adherence measures, the study populations, study durations, and the exact outcome measures varied.

\section{Insulin Studies}

A total of 18 published articles (including Levin et al. [32]) reported relationships between insulin adherence or persistence and HbA1c. Of these 18,5 studies $[5,17,19,21,32]$ examined 
Table 3 Studies regarding the impact of adherence or persistence on glycemic control $(n=25)$

\begin{tabular}{|c|c|c|c|}
\hline Author & $\begin{array}{l}\text { Medication and study } \\
\text { population }\end{array}$ & Adherence/persistence & $\begin{array}{l}\text { Glycemic control relationship to adherence } \\
\text { or persistence }\end{array}$ \\
\hline Ivanyi [38] & $\begin{array}{l}\text { GLP-1 RA, naïve, initiators } \\
\text { on metformin and/or } \\
\text { SFU }\end{array}$ & Persistence: 132 weeks & $\begin{array}{l}\text { Change in } \mathrm{HbAlc} \text { from baseline (mean }[\mathrm{SD}] \\
\quad(P<0.0001 \text { at all time points) } \\
\text { Persisters: week } 52:-1.3 \%(0.10 \%) \\
\text { Persisters: week } 100:-1.0 \%(0.12 \%) \\
\text { Persisters: week } 132:-1.0 \%(0.13 \%)\end{array}$ \\
\hline Varanasi [37] & $\begin{array}{l}\text { GLP-1 RA, naïve, initiators } \\
\text { on oral medication with } \\
\text { or without insulin }\end{array}$ & Persistence: 6 weeks & $\begin{array}{l}\text { Change in } \mathrm{HbAlc} \text { from baseline (mean }[\mathrm{SD}] \\
\text { Persisters: week 6: drop from } 8.2 \%(0.5 \%) \text { to } \\
6.9 \%(0.4 \%), P<0.001 \\
\text { Nonpersisters: at stop: drop from } 7.7 \% \\
(0.6 \%) \text { to } 7.2 \%(0.3 \%), P<0.001 \\
\text { At } 6 \text { months after stop: rise to } 7.4 \%(0.2 \%) \\
P=0.04 \text { compared to value at stop }\end{array}$ \\
\hline $\begin{array}{l}\text { Buysschaert } \\
\text { [36] }\end{array}$ & $\begin{array}{l}\text { GLP-1 RA, naïe, initiators } \\
\text { on metformin and SFU }\end{array}$ & Persistence: 1 year & $\begin{array}{l}\text { Change in HbAlc from baseline (mean) } \\
(P \text { values not reported }) \\
\text { Persisters: }-1.0 \%(8.1 \% \text { to } 7.2 \%) \text { when } \\
\text { baseline HbAlc was } \leq 9 \% \\
\text { Persisters: }-2.4 \%(10.2 \% \text { to } 7.8 \%) \text { when } \\
\text { baseline HbAlc was }>9 \% \\
\text { Nonpersisters: }+0.5 \%(8.4 \% \text { to } 8.9 \%) \text { when } \\
\text { baseline HbAlc was } \leq 9 \% \\
\text { Nonpersisters: }-0.6 \%(10.6 \% \text { to } 10.0 \%) \\
\text { when baseline HbAlc was }>9 \%\end{array}$ \\
\hline Levin [32] & $\begin{array}{l}\text { GLP-1 RA, naïve, initiators } \\
\text { as third agent to two } \\
\text { prior oral medications }\end{array}$ & Persistence: 2 years & $\begin{array}{l}\text { Change in HbAlc from baseline (mean) } \\
\text { (None is statistically significant) } \\
\text { Persisters years } 1 \text { and } 2:-0.48 \% \\
\text { Persisters year } 1 \text { with switch year 2: }-0.27 \% \\
\text { Switched year 1: }-0.23 \% \\
\text { Discontinued (not filling any diabetes drug in } \\
\text { last quarter of year } 1 \text { or year 2): }-0.38 \%\end{array}$ \\
\hline $\operatorname{Lin}[31]$ & $\begin{array}{l}\text { Initiators of combination } \\
\text { of GLP-1 RA and insulin }\end{array}$ & Persistence: 1 year & $\begin{array}{l}\text { Change in } \mathrm{HbAlc} \text { from baseline (mean) } \\
\text { Persisters vs nonpersisters: }-0.8 \% \text { vs }-0.4 \% \\
P=0.032\end{array}$ \\
\hline
\end{tabular}


Table 3 continued

\begin{tabular}{|c|c|c|c|}
\hline Author & $\begin{array}{l}\text { Medication and study } \\
\text { population }\end{array}$ & Adherence/persistence & $\begin{array}{l}\text { Glycemic control relationship to adherence } \\
\text { or persistence }\end{array}$ \\
\hline Buysman [34] & $\begin{array}{l}\text { GLP-1 RA, naive, initiators } \\
\text { on oral medications and/ } \\
\text { or insulin }\end{array}$ & $\begin{array}{l}\text { Adherence and } \\
\text { persistence: } 1 \text { year }\end{array}$ & $\begin{array}{l}\text { Odds ratio for adherent vs nonadherent at } \\
1 \text { year } \\
\text { PDC } \geq 80 \% \text { with HbA1c goal }<7.0 \% \text { : OR } \\
1.84, P<0.001 \text { ( } 51 \% \text { vs } 39 \%) \\
\text { PDC } \geq 80 \% \text { with HbAlc goal }<6.5 \% \text { : OR } \\
1.70, P<0.001 \text { ( } 35 \% \text { vs } 26 \%) \\
\text { Reduction HbAlc } \geq 1.0 \% \text {, with } \\
\text { PDC } \geq 80 \% \text { : OR } 1.86, P<0.001(41 \% \text { vs } \\
30 \%) \\
\text { Odds ratio for persistent vs nonpersistent at } \\
1 \text { year } \\
\text { With HbA1c goal }<7.0 \% \text { : OR } 2.34, \\
P<0.001(34 \% \text { vs } 23 \%) \\
\text { With HbAlc goal }<6.5 \% \text { OR } 2.01, \\
P<0.001 \text { ( } 49 \% \text { vs } 33 \%) \\
\text { Reduction in HbAlc } \geq 1.0 \% \text { with } \\
\text { persistence: OR } 2.37(P<0.001)(39 \% \text { vs } \\
26 \%)\end{array}$ \\
\hline Durden [33] & $\begin{array}{l}\text { GLP-1 RA, naïve, initiators } \\
\text { with or without prior } \\
\text { oral medication }\end{array}$ & Adherence: 6 months & $\begin{array}{l}\text { Odds ratio for adherent }(\mathrm{PDC} \geq 80 \%) \text { vs } \\
\text { nonadherent to have } \mathrm{HbA} 1 \mathrm{c}<7 \% \text { at } \\
6 \text { months } \\
\text { OR 1.83, 95\%CI 1.11-3.01, } P<0.05\end{array}$ \\
\hline Carls [35] & $\begin{array}{l}\text { GLP-1 RA, naïve, initiators } \\
\text { with prior oral } \\
\text { medications }\end{array}$ & Adherence: 1 year & $\begin{array}{l}\text { Change in HbAlc from baseline (mean) } \\
(P \text { values not reported }) \\
\text { PDC } \geq 80 \% \text { vs PDC < } 80 \% \text { : }-0.86 \% \text { vs } \\
-0.39 \% \\
\text { For every 1-point increase in baseline HbA1c } \\
\text { levels, final HbA1c decreased by an } \\
\text { additional } 0.275 \% \\
\text { Medication adherence accounted for } \sim 75 \% \\
\text { of the estimated } 0.41 \% \text { HbAlc gap between } \\
\text { real-world and randomized controlled trial } \\
\text { results for patients receiving GLP-1 RA } \\
\text { therapy }\end{array}$ \\
\hline
\end{tabular}


Table 3 continued

\begin{tabular}{|c|c|c|c|}
\hline Author & $\begin{array}{l}\text { Medication and study } \\
\text { population }\end{array}$ & Adherence/persistence & $\begin{array}{l}\text { Glycemic control relationship to adherence } \\
\text { or persistence }\end{array}$ \\
\hline $\mathrm{Wu}[17]$ & $\begin{array}{l}\text { Insulin, non-naïve, upon } \\
\text { discharge from hospital }\end{array}$ & Persistence: 1 year & $\begin{array}{l}\text { Change in HbAlc from baseline (mean) } \\
\text { Persisters vs nonpersisters: }-0.5 \% \text { vs }-0.2 \% \text {, } \\
P<0.001\end{array}$ \\
\hline Wei [5] & $\begin{array}{l}\text { Insulin, naïve, } \\
\text { receiving }>1 \text { oral } \\
\text { medication or GLP-1 RA }\end{array}$ & Persistence: 1 year & $\begin{array}{l}\text { Change in HbAlc from baseline (mean) } \\
\text { Persisters vs nonpersisters: }-1.2 \% \text { vs }-0.9 \% \text {, } \\
P=0.0078\end{array}$ \\
\hline $\begin{array}{l}\text { Sambamoorthi } \\
\quad[19]\end{array}$ & $\begin{array}{l}\text { Basal insulin, with addition } \\
\text { of rapid-acting insulin }\end{array}$ & Persistence: 1 year & $\begin{array}{l}\text { Change in } \mathrm{HbAlc} \text { from baseline (mean }[\mathrm{SD}] \text { ) } \\
\text { in elderly patients with low persistence rate } \\
(21 \%) \\
\text { Persisters vs nonpersisters: }-0.8 \%(1.55 \%) \text { vs } \\
-0.4 \%(1.41 \%) P<0.01\end{array}$ \\
\hline Yavuz [21] & $\begin{array}{l}\text { Insulin, naïve, initiated on } \\
\text { pen therapy }\end{array}$ & Persistence: 6 months & $\begin{array}{l}\text { HbAlc (mean }[\mathrm{SD}]) \text { at end of study } \\
\text { Total population baseline: } 11.2 \%(1.5 \%) \\
\text { Persisters vs nonpersisters: } 10.1 \%(1.8 \%) \text { vs } \\
10.4 \%(2.0 \%), P=0.4\end{array}$ \\
\hline Levin [32] & $\begin{array}{l}\text { Insulin, naïve, initiated as } \\
\text { third agent }\end{array}$ & Persistence: 2 years & $\begin{array}{l}\text { Change in HbAlc from baseline (mean) } \\
(P \text { values not reported }) \\
\text { Persisters years } 1 \text { and } 2:-0.99 \% \\
\text { Persisters year } 1 \text { with switch year } 2:-0.93 \% \\
\text { Switched year } 1:-0.59 \%(P<0.05 \text {, when } \\
\text { compared with persisters years } 1 \text { and } 2) \\
\text { Discontinued (not filling any diabetes drug in } \\
\text { last quarter of year } 1 \text { or year } 2):-0.97 \%\end{array}$ \\
\hline Ayyagari [15] & $\begin{array}{l}\text { Insulin, naïve, previously } \\
\text { on oral medication or } \\
\text { GLP-1 RA }\end{array}$ & Adherence: 1 year & $\begin{array}{l}\text { Adjusted change in HbAlc from baseline } \\
\text { (mean) } \\
\text { Adherers vs nonadherers: greater mean } \\
\text { decrease in HbAlc from baseline when using } \\
\text { same type of device, but differences within } \\
\text { device type not statistically significant } \\
\text { Vial: }-0.2 \%(P=0.138) \\
\text { Pen: }-0.2 \%(P=0.138)\end{array}$ \\
\hline Egede [11] & $\begin{array}{l}\text { Insulin, non-naïve, in a } \\
\text { cohort based on calendar } \\
\text { year of study period }\end{array}$ & $\begin{array}{l}\text { Adherence: } 12 \text { years, } \\
\text { measured at } 3 \text {-month } \\
\text { intervals over time }\end{array}$ & $\begin{array}{l}\text { Adjusted change in } \mathrm{HbAlc} \text { from baseline for } \\
\text { insulin-only patients ( } P \text { value not reported) } \\
0.05 \% \text { for each percentage increase in MPR }\end{array}$ \\
\hline
\end{tabular}


Table 3 continued

\begin{tabular}{|c|c|c|c|}
\hline Author & $\begin{array}{l}\text { Medication and study } \\
\text { population }\end{array}$ & Adherence/persistence & $\begin{array}{l}\text { Glycemic control relationship to adherence } \\
\text { or persistence }\end{array}$ \\
\hline Donnelly [16] & $\begin{array}{l}\text { Insulin, non-naïve, in a } \\
\text { cohort based on calendar } \\
\text { year of study period }\end{array}$ & Adherence: 6 years & $\begin{array}{l}\text { Change in HbAlc from baseline } \\
\text { PDC } \geq 80 \% \text { were more likely to } \\
\text { demonstrate improved HbAlc } \\
\text { Significant inverse association between log } \\
\text { adherence and HbAlc }(P<0.0001) \\
\text { HbAlc increased over time, demonstrating a } \\
\text { significant quadratic trend ( } P=0.0023), \\
\text { accompanied by a significant linear trend } \\
(P<0.0001) \\
\text { In addition there was a significant } \\
\text { interaction between adherence and time, } \\
\text { suggesting longitudinal changes in HbAlc } \\
\text { varied according to adherence }(P<0.0001)\end{array}$ \\
\hline Kindmalm [20] & $\begin{array}{l}\text { Insulin, non-naïve, in a } \\
\text { cohort based on calendar } \\
\text { year of study period }\end{array}$ & Adherence: 1 year & $\begin{array}{l}\text { HbAlc at end of study period } \\
\text { Refill adherence } \geq 80 \% \text { had a lower mean } \\
\text { HbAlc }(6.6 \% \text { vs } 7.3 \%)(P=0.025)\end{array}$ \\
\hline Linetsky $[10]$ & $\begin{array}{l}\text { Insulin, non-naïve, in a } \\
\text { cohort based on calendar } \\
\text { year of study period }\end{array}$ & Adherence: baseline data & $\begin{array}{l}\text { Cross-sectional measurement of HbAlc at } \\
\text { baseline } \\
\text { Patients with "missed shots" had a } 0.43 \% \\
\text { higher HbAlc in path analysis }\end{array}$ \\
\hline Linetsky [22] & $\begin{array}{l}\text { Insulin, non-naïve, in a } \\
\text { cohort based on calendar } \\
\text { year of study period }\end{array}$ & Adherence: baseline data & $\begin{array}{l}\text { Change in HbAlc from baseline (mean, } \\
95 \% \mathrm{CI}) \\
\text { Patients with "no missed shots" had a lower } \\
\text { HbAlc in multivariate linear regression } \\
-0.19 \%(-0.34 \text { to }-0.05), P=0.0104\end{array}$ \\
\hline Aikens [24] & $\begin{array}{l}\text { Insulin, non-naïve, in a } \\
\text { cohort based on calendar } \\
\text { year of study period }\end{array}$ & Adherence: 6 months & $\begin{array}{l}\text { Cross-sectional measurement of adherence at } \\
\text { baseline and HbAlc at end of study period } \\
\text { 1-unit increase in 4-unit Morisky score } \\
\text { (nonadherence) associated with a } 0.16 \% \\
\text { increase in HbAlc ( } P \text { value not reported) }\end{array}$ \\
\hline Osborn [12] & $\begin{array}{l}\text { Insulin, non-naïve, in a } \\
\text { cohort based on calendar } \\
\text { year of study period }\end{array}$ & $\begin{array}{l}\text { Adherence: at time of } \\
\text { HbAlc measurement }\end{array}$ & $\begin{array}{l}\text { Cross-sectional measurement of HbAlc at } \\
\text { baseline } \\
\text { Increase in 4-unit modified Morisky score } \\
\text { (adherence) (modified for insulin use- } \\
\text { MIAS) associated with a decrease in HbAlc } \\
(-0.26 \%, P=0.001 \text { ) }\end{array}$ \\
\hline
\end{tabular}


Table 3 continued

\begin{tabular}{|c|c|c|c|}
\hline Author & $\begin{array}{l}\text { Medication and study } \\
\text { population }\end{array}$ & Adherence/persistence & $\begin{array}{l}\text { Glycemic control relationship to adherence } \\
\text { or persistence }\end{array}$ \\
\hline Mashitani [18] & $\begin{array}{l}\text { Insulin, non-naïve, in a } \\
\text { cohort based on calendar } \\
\text { year of study period }\end{array}$ & $\begin{array}{l}\text { Adherence: at time of } \\
\text { HbAlc measurement }\end{array}$ & $\begin{array}{l}\text { Cross-sectional measurement of HbAlc at } \\
\text { baseline } \\
\text { Compared to participants with higher } \\
\text { adherence, the crude } \mathrm{RR}^{\mathrm{j}} \text { for good glycemic } \\
\text { control (HbAlc }<7.0 \%) \text { for those with } \\
\text { middle adherence was } 0.82(0.67-1.00) \text {, and } \\
\text { for lower adherence } 0.64(0.31-1.31) \\
(P=0.029 \text { for trend) }\end{array}$ \\
\hline Nishimura [23] & $\begin{array}{l}\text { Insulin glargine, non-naïe, } \\
\text { switching to insulin } \\
\text { degludec }\end{array}$ & Adherence: 12 weeks & $\begin{array}{l}\text { Cross-sectional measurement of HbAlc at } \\
\text { baseline (mean }[\mathrm{SE}]) \\
\text { Timing of shots: higher HbAlc with longer } \\
\text { delays }(P>0.05) \\
0-120 \text { min delayed dosing: } 8.2 \%(1.2 \%) \text { to } \\
8.1 \%(1.0 \%), P=\mathrm{NS} \\
121-240 \text { min delayed dosing: switch caused } \\
\text { HbAlc change from } 8.0 \%(1.2 \%) \text { to } 7.7 \% \\
(1.3 \%), P=0.039\end{array}$ \\
\hline $\begin{array}{l}\text { Perez-Nieves } \\
\text { [9] }\end{array}$ & $\begin{array}{l}\text { Insulin, naïve, initiators of } \\
\text { basal insulin }\end{array}$ & $\begin{array}{l}\text { Continuers vs interrupters } \\
\text { vs discontinuers } \\
\text { (identified } \\
\text { retrospectively) }\end{array}$ & $\begin{array}{l}\text { Cross-sectional survey } \\
\text { Continuers were more likely to report a } \\
\text { positive impact of insulin on glycemic } \\
\text { control compared to interrupters and } \\
\text { discontinuers ( } 73.0 \% \text { vs } 63.0 \% \text { vs } 61.8 \% \text {, } \\
P<0.01 \text { vs continuers) }\end{array}$ \\
\hline $\begin{array}{l}\text { Dibonaventura } \\
\text { [13] }\end{array}$ & $\begin{array}{l}\text { Insulin, non-naïe, in a } \\
\text { cohort based on calendar } \\
\text { year of study period }\end{array}$ & $\begin{array}{l}\text { Adherence: at time of } \\
\text { HbAlc report }\end{array}$ & $\begin{array}{l}\text { Cross-sectional survey } \\
\text { Each point increase in 4-unit Morisky score } \\
\text { (nonadherence) was associated with a } 0.21 \% \\
\text { increase in } \mathrm{HbAlc}(P<0.05)\end{array}$ \\
\hline
\end{tabular}


Table 3 continued

\begin{tabular}{|c|c|c|c|}
\hline Author & $\begin{array}{l}\text { Medication and study } \\
\text { population }\end{array}$ & Adherence/persistence & $\begin{array}{l}\text { Glycemic control relationship to adherence } \\
\text { or persistence }\end{array}$ \\
\hline Leiter [6] & $\begin{array}{l}\text { Insulin, perspectives of } \\
\text { healthcare providers }\end{array}$ & Adherence & $\begin{array}{l}\text { Cross-sectional survey regarding healthcare } \\
\text { providers' assessment of the number of } \\
\text { occasions of dosing irregularities in } 30 \text {-day } \\
\text { period that would impact glycemic control } \\
3.6 \text { for missed doses } \\
5.7 \text { for mistimed doses } \\
4.1 \text { for reduced doses } \\
39 \% \text { of healthcare providers reported not } \\
\text { discussing dosing irregularities with patients }\end{array}$ \\
\hline
\end{tabular}

Levin et al. [32] is represented twice because it reports regarding two different drug regimens

GLP-1 RA glucose-like peptide-1 receptor agonist, $S F U$ sulfonylurea, $S D$ standard deviation, $P D C$ proportion of days covered, $O R$ odds ratio, $C I$ confidence interval, $M P R$ medication possession ratio, $R R$ relative risk, $S E$ standard error, $H b A 1 c$ hemoglobin Alc

persistence and 13 examined adherence. Here too, study designs varied widely, precluding any evaluation of trends or synthesis of study results. See Table 3 for individual study details.

The five studies examining persistence were all retrospective cohort studies that examined changes in HbA1c over time. At 1-year, reports of persistence in these five studies ranged from 21 to $66 \%$. They all demonstrated some drop in HbA1c from baseline, although 1 study [32] did not show statistical significance. Study populations differed with respect to enrolling continuing or naïve insulin users (and additional medications used by study participants), and study durations ranged from 6 months to 2 years.

Of the remaining 13 insulin studies, 4 examined adherence over time in retrospective cohorts $[11,15,16,20]$, and 9 examined adherence and $\mathrm{HbA} 1 \mathrm{c}$ in cross-sectional studies, many of which involved patient interviews. Adherence to insulin regimens was generally associated with lower HbA1c, but 3 studies did not document statistically significant differences $[10,11,24]$. Several studies attempted to quantify the association between the degree of adherence and HbA1c [10, 11, 13, 16, 18, 22-24], although these were generally not high-quality studies.

\section{HEALTHCARE RESOURCE USE RESULTS}

\section{Studies Regarding the Impact of Adherence and Persistence on Medical Resource Use}

The 9 articles identified included 2 cross-sectional studies $[12,13]$ and 7 studies using administrative/claims data $[5,7,15,17,25,29,31]$, of which 2 were supplemented with electronic medical record (EMR) [17] or laboratory [15] data. Only 1 study included GLP-1 RA patients (exenatide or liraglutide), and these patients initiated combination therapy with insulin [31].

\section{Improvements in Healthcare Resource Use}

Adherence and persistence appeared to have an inverse relationship with healthcare resource use. Studies consistently found a reduction in rates of all-cause and diabetes-related hospital, emergency department (ED) visits and inpatient days; most studies also found a reduction in outpatient visits in adherent/persistent patients. 


\section{GLP-1 RA Studies}

The only study that included GLP-1 RA medication was Lin et al. [31]. One-year persistence after initiating a combination treatment with insulin and GLP-1 RA was associated with improvements in all-cause and diabetes-related hospitalizations and length of stay.

\section{Insulin Studies}

Ayyagari et al. [15] demonstrated that adherence was associated with fewer hospitalizations in both pen and syringe/vial users. Pen users had increased adherence. Two other lesser quality studies examined adherence. Osborn et al. [12] found that self-reported adherence was associated with fewer ED visits, and DiBonaventura et al. [13] noted that self-reported adherence was associated with fewer ED, physician, and inpatient visits.

Wei et al. [5] found that persistence was associated with fewer all-cause ED, all-cause hospitalization, and diabetes-related hospitalization visits. Perez-Nieves et al. [29] observed that medication persistence was associated with fewer all-cause ED visits, diabetes-related ED visits, all-cause hospitalization days, diabetesrelated hospitalization days, all-cause outpatient visits, at least 1 fill of premixed or nonbasal insulin, and 1 fill of any non-insulin antihyperglycemic injectable.

$\mathrm{Wu}$ et al. [17] recounted that patients who continued insulin therapy after hospital discharge had lower risks of all-cause hospital readmission, diabetes-related hospital readmission, and all-cause ED visits. Twelve months after hospital discharge, the insulin therapy continuation group had a higher all-cause hospital readmission-free rate and a higher allcause ED visit-free rate. Diabetes-related hospital readmission-free rates and diabetes-related ED visit-free rates were also significantly higher among patients who continued therapy.

Hadjiyianni et al. [25] noted that all-cause inpatient days were reduced for continuers compared with interrupters and discontinuers; however, all-cause outpatient visits were not.
Wang et al. [7] showed that persistence was associated with fewer hospitalizations.

\section{HEALTHCARE COSTS RESULTS}

\section{Studies Regarding the Impact of Adherence and Persistence on Healthcare Costs}

Of the 14 articles identified, 11 used administrative/claims data $[1,8,15,17,25,26,28-31,34]$ (of which two were supplemented with EMR [17] or laboratory [15] data), 2 used an employee or healthcare provider database $[14,27]$, and 1 used a crosssectional design [9]. Only 2 [31, 34] included GLP-1 RA patients.

\section{Improvements in Costs}

The literature largely showed positive relationships between adherence or persistence measures and lower healthcare costs in T2DM subjects. However, for total all-cause and diabetes-related healthcare costs, the increase in pharmacy costs for adherent/persistent patients tended to partially or completely offset the gains in other healthcare costs (Table 4).

\section{GLP-1 RA Studies}

Two studies included GLP-1 RA medications $[31,34]$. Buysman et al. [34] considered 1-year adherence and persistence. Although diabetesrelated pharmacy and total healthcare costs were higher for the adherent and persistent groups, these patients incurred significantly lower diabetes-related medical costs (Table 4).

Lin et al. [31] examined patients initiating a combination therapy of insulin and GLP-1 RA and grouped patients by 1-year persistence with both medications. In adjusted regression models, persistence with combination therapy was a predictor of lower all-cause inpatient and outpatient medical costs during the follow-up period (estimate [95\% CI] - 0.24 [- 0.43, - 0.05]; $P=0.013)$, and a predictor of lower diabetesrelated inpatient and outpatient medical costs 
Table 4 Studies regarding the impact of adherence or persistence on healthcare costs $(n=14)$

\begin{tabular}{|c|c|c|c|}
\hline Author & $\begin{array}{l}\text { Medication and study } \\
\text { population }\end{array}$ & Adherence/persistence & $\begin{array}{l}\text { Costs for adherent/persistent vs } \\
\text { nonadherent/nonpersistent groups }\end{array}$ \\
\hline \multirow[t]{16}{*}{$\begin{array}{l}\text { Buysman } \\
\text { [34] }\end{array}$} & \multirow{16}{*}{$\begin{array}{l}\text { GLP-1 RA, naïve, initiators on } \\
\text { oral medications and/or } \\
\text { insulin }\end{array}$} & \multirow[t]{4}{*}{$\begin{array}{l}\text { Adherence and } \\
\text { persistence: } 1 \text { year }\end{array}$} & $\begin{array}{l}\text { Diabetes-related, unadjusted (mean }[\mathrm{SD}] \text {; } \\
\text { median) }\end{array}$ \\
\hline & & & $\begin{array}{l}\text { Total: } \$ 9,081(\$ 8685) ; \$ 6797 \text { vs } \$ 7717 \\
(\$ 13,679) ; \$ 4647, P=0.028\end{array}$ \\
\hline & & & $\begin{array}{l}\text { Pharmacy total: } \$ 6338(\$ 2,639) ; \$ 5606 \text { vs } \\
\$ 3568 \text { (\$2439); } \$ 3074, P<0.001\end{array}$ \\
\hline & & & $\begin{array}{l}\text { Total medical: } \$ 2743(\$ 8,065) ; \$ 683 \text { vs } \\
\$ 4149(\$ 13,383) ; \$ 687, P=0.018\end{array}$ \\
\hline & & \multirow[t]{4}{*}{ Adherence: 1 year } & Diabetes-related, adjusted \\
\hline & & & $\begin{array}{l}\text { Total }(95 \% \mathrm{CI}): \$ 9419(\$ 8574-10,308) \text { vs } \\
\$ 7667(\$ 6903-8573), P=0.005\end{array}$ \\
\hline & & & $\begin{array}{l}\text { Pharmacy total (estimated from figure): } \\
\$ 6000 \text { vs } \$ 4000, P<0.001\end{array}$ \\
\hline & & & $\begin{array}{l}\text { Total medical (estimated from figure): } \$ 3000 \\
\text { vs } \$ 4000, P=0.080\end{array}$ \\
\hline & & \multirow[t]{4}{*}{ Persistence: 1 year } & $\begin{array}{l}\text { Diabetes-related, unadjusted (mean [SD]; } \\
\text { median) }\end{array}$ \\
\hline & & & $\begin{array}{l}\text { Total: } \$ 8675(\$ 10,611) ; \$ 6180 \text { vs } \$ 7447 \\
(\$ 14,270) ; \$ 3864, P=0.092\end{array}$ \\
\hline & & & $\begin{array}{l}\text { Pharmacy total: } \$ 5571(\$ 2658) ; \$ 5039 \text { vs } \\
\$ 2931(\$ 2298) ; \$ 2341, P<0.001\end{array}$ \\
\hline & & & $\begin{array}{l}\text { Total medical: } \$ 3103(\$ 10,124) ; \$ 682 \text { vs } \\
\$ 4516(\$ 14,017) ; \$ 699, P=0.047\end{array}$ \\
\hline & & \multirow[t]{4}{*}{ Persistence: 1 year } & Diabetes-related, adjusted (mean) \\
\hline & & & $\begin{array}{l}\text { Total (estimated from figure): } \$ 8700 \text { vs } \\
\$ 7500, P=0.010\end{array}$ \\
\hline & & & $\begin{array}{l}\text { Pharmacy total (estimated from figure): } \\
\$ 5000 \text { vs } \$ 3500, P=0.010\end{array}$ \\
\hline & & & $\begin{array}{l}\text { Total medical (stated in text): } \$ 3298 \text { vs } \\
\$ 4805, P=0.017\end{array}$ \\
\hline
\end{tabular}


Table 4 continued

\begin{tabular}{|c|c|c|c|}
\hline Author & $\begin{array}{l}\text { Medication and study } \\
\text { population }\end{array}$ & Adherence/persistence & $\begin{array}{l}\text { Costs for adherent/persistent vs } \\
\text { nonadherent/nonpersistent groups }\end{array}$ \\
\hline $\operatorname{Lin}[31]$ & $\begin{array}{l}{ }^{\text {a} I n i t i a t o r s ~ o f ~ c o m b i n a t i o n ~ o f ~} \\
\text { GLP-1 RA and insulin }\end{array}$ & Persistence: 1 year & $\begin{array}{l}\text { All-cause, unadjusted } \\
\text { Total: } \$ 43,096 \text { vs } \$ 51,084, P=0.028 \\
\text { Pharmacy total: } \$ 14,691 \text { vs } \$ 10,791 \text {, } \\
P<0.001 \\
\text { Total medical: } \$ 28,405 \text { vs } \$ 40,292 \text {, } \\
P=0.001\end{array}$ \\
\hline & & Persistence: 1 year & $\begin{array}{l}\text { Diabetes-related, unadjusted } \\
\text { Total: } \$ 19,255 \text { vs } \$ 20,327, P=0.441 \\
\text { Pharmacy total: } \$ 8142 \text { vs } \$ 5124, P<0.001 \\
\text { Total medical: } \$ 11,114 \text { vs } \$ 15,203 \text {, } \\
P=0.003\end{array}$ \\
\hline Kalirai [30] & Insulin, naïve, initiators & Persistence: 1 and 2 years & $\begin{array}{l}\text { Year 1: Adjusted estimates from regression } \\
\text { models } \\
\text { All-cause total medical, inpatient and ED } \\
\text { costs were significantly lower, but not } \\
\text { outpatient costs } \\
\text { Diabetes related total medical, inpatient and } \\
\text { ED costs were significantly lower, but not } \\
\text { outpatient costs } \\
\text { Diabetes-related pharmacy basal and other } \\
\text { insulins and total pharmacy costs were } \\
\text { significantly higher, but this was not true for } \\
\text { other injectables and oral antidiabetic } \\
\text { medications } \\
\text { All-cause healthcare (medical }+ \text { pharmacy) } \\
\text { costs were nonsignificantly lower for } \\
\text { continuers } \\
\text { Year } 2: \text { Results were similar with the } \\
\text { exception that diabetes-related pharmacy } \\
\text { costs for injectables were also significantly } \\
\text { higher for continuers }\end{array}$ \\
\hline
\end{tabular}


Table 4 continued

\begin{tabular}{|c|c|c|c|}
\hline Author & $\begin{array}{l}\text { Medication and study } \\
\text { population }\end{array}$ & Adherence/persistence & $\begin{array}{l}\text { Costs for adherent/persistent vs } \\
\text { nonadherent/nonpersistent groups }\end{array}$ \\
\hline $\begin{array}{l}\text { Ascher- } \\
\text { Svanum } \\
{[8]}\end{array}$ & Insulin, naïve, initiators & $\begin{array}{l}\text { Early discontinuation } \\
\text { (gap } \geq 30 \text { days after } \\
\text { first prescription): } \\
1 \text { year }\end{array}$ & $\begin{array}{l}\text { Adjusted estimates from regression models, all } \\
\qquad P \text { values }<0.05 \\
\text { Acute care costs (inpatient }+ \text { ED): } 9.6 \% \\
\text { higher } \\
\text { Outpatient costs: } 6.4 \% \text { lower } \\
\text { Diabetes-related pharmacy costs: } 42.9 \% \\
\text { lower } \\
\text { All-cause pharmacy costs: } 34.0 \% \text { lower } \\
\text { Total medical costs: } 10.9 \% \text { lower }\end{array}$ \\
\hline $\mathrm{Wu}[17]$ & $\begin{array}{l}\text { Insulin, non-naïve, upon } \\
\text { discharge from hospital }\end{array}$ & Persistence: 6 months & $\begin{array}{l}\text { Regression models with adjustment for pre- } \\
\text { discharge costs and other baseline covariates } \\
\text { Lower total medical service costs (difference } \\
\text { of } \$ 2569, P=0.007 \text { ) } \\
\text { Results driven by lower facility and other } \\
\text { services costs (difference of } \$ 2265 \text {; } \\
P=0.010 \text { ), and physician service costs } \\
\text { (difference of } \$ 524 ; P=0.001 \text { ) (pharmacy } \\
\text { costs not reported) }\end{array}$ \\
\hline $\begin{array}{l}\text { Perez-Nieves } \\
\text { [29] }\end{array}$ & $\begin{array}{l}\text { Insulin, naive, initiators of non- } \\
\text { mixed basal insulin during } \\
\text { study period }\end{array}$ & $\begin{array}{l}\text { Continuers vs interrupters } \\
\text { vs discontinuers }\end{array}$ & $\begin{array}{l}\text { Year } 1 \\
\text { Continuers had lower medical costs } \\
\text { (continuers: } \$ 10,890 \text {, interrupters: } \$ 13,674 \text {, } \\
\text { discontinuers } \$ 13,021 \\
\text { Continuers had higher pharmacy costs } \\
\text { (continuers: } \$ 7449 \text {, interrupters: } \$ 5239 \text {, } \\
\text { discontinuers } \$ 4857 \\
P<0.05 \text { for all comparisons of continuers } \\
\text { vs interrupters and continuers vs } \\
\text { discontinuers } \\
\text { Total healthcare costs similar across the } 3 \\
\text { cohorts } \\
\text { Year } 2 \text { : findings were similar }\end{array}$ \\
\hline $\begin{array}{l}\text { Anderten } \\
\quad[14]\end{array}$ & $\begin{array}{l}\text { Insulin, naive, initiators of basal } \\
\text { insulin }\end{array}$ & Persistence: 1 year & $\begin{array}{l}\text { No differences in treatment or prescription } \\
\text { costs or medical services by persistence to } \\
\text { insulin glargine vs NPH }\end{array}$ \\
\hline
\end{tabular}


Table 4 continued

\begin{tabular}{|c|c|c|c|}
\hline Author & $\begin{array}{l}\text { Medication and study } \\
\text { population }\end{array}$ & Adherence/persistence & $\begin{array}{l}\text { Costs for adherent/persistent vs } \\
\text { nonadherent/nonpersistent groups }\end{array}$ \\
\hline $\begin{array}{l}\text { Kleinman } \\
\quad[27]\end{array}$ & Insulin, naive, initiators & Adherence (MPR): 1 year & $\begin{array}{l}\text { Regression models stratified by high vs low } \\
\text { prior costs } \\
\text { Higher MPR was associated with } \\
\text { significantly lower total healthcare costs for } \\
\text { patients with high (upper quartile) prior } \\
\text { costs: } \$ 450 \text { in savings per } 10 \% \text { increase in } \\
\text { MPR } \\
\text { Higher MPR was associated with } \\
\text { significantly higher total healthcare costs for } \\
\text { patients with low (lower quartile) prior costs } \\
\text { (amount not stated) } \\
\text { Both groups of patients experienced } \\
\text { significantly lower medical costs (removing } \\
\text { prescription costs) when MPR was high } \\
\text { Higher prior cost group: } 100 \% \text { MPR group } \\
\text { had } \$ 6,653 \text { in medical costs vs } 10 \% \text { MPR } \\
\text { group had } \$ 11,763 \text { in medical costs } \\
\text { Lower prior cost group: } 100 \% \text { MPR group } \\
\text { had } \$ 3329 \text { in medical costs vs } 10 \% \text { MPR } \\
\text { group had } \$ 4,590 \text { in medical costs }\end{array}$ \\
\hline $\begin{array}{c}\text { Ayyagari } \\
{[15]}\end{array}$ & $\begin{array}{l}\text { Insulin, naïve, previously on } \\
\text { oral medication or GLP-1 } \\
\text { RA }\end{array}$ & Adherence: 1 year & $\begin{array}{l}\text { Adjusted costs using marginal structural } \\
\text { models } \\
\text { Pharmacy costs higher for adherent insulin } \\
\text { users, averaging an additional } \$ 2,074 \text { more } \\
\text { annually for pen users and } \$ 2923 \text { more for } \\
\text { vial users ( } P<0.001 \text { for both patterns) } \\
\text { Annual health care costs for both pen and } \\
\text { vial users demonstrated lower costs for } \\
\text { nonadherent patients, but these differences } \\
\text { were not statistically significant }\end{array}$ \\
\hline $\begin{array}{c}\text { Cobden } \\
\text { [26] }\end{array}$ & $\begin{array}{l}\text { Insulin, continuing, who } \\
\text { converted from an insulin } \\
\text { analog or human insulin } \\
\text { administered using a vial/ } \\
\text { syringe to a biphasic insulin } \\
\text { analog administered with a } \\
\text { pen device }\end{array}$ & $\begin{array}{l}\text { Adherence } \\
\qquad(\mathrm{MPR} \geq 80 \%): 2 \text { years }\end{array}$ & $\begin{array}{l}\text { Adjusted costs } \\
\text { Adherence was associated with significant } \\
\text { reductions in all-cause healthcare costs } \\
\text { (exponentiated coefficient estimate } 0.55 \text {, } \\
95 \% \text { CI } 0.31-0.80, P<0.05 \text { ). This implies } \\
\text { an average } 45 \% \text { decrease in all-cause total } \\
\text { healthcare costs for adherent compared to } \\
\text { nonadherent patients }\end{array}$ \\
\hline
\end{tabular}


Table 4 continued

\begin{tabular}{|c|c|c|c|}
\hline Author & $\begin{array}{l}\text { Medication and study } \\
\text { population }\end{array}$ & Adherence/persistence & $\begin{array}{l}\text { Costs for adherent/persistent vs } \\
\text { nonadherent/nonpersistent groups }\end{array}$ \\
\hline Wei [5] & $\begin{array}{l}\text { Insulin, naïe, receiving }>1 \\
\text { oral medication or GLP-1 } \\
\text { RA }\end{array}$ & Persistence: 1 year & $\begin{array}{l}\text { Unadjusted costs } \\
\text { Pharmacy costs: } \$ 5761 \text { vs } \$ 4319, P<0.0001 \\
\text { Total medical costs: } \$ 17,007 \text { vs } \$ 18,367 \text {, } \\
P=0.1419\end{array}$ \\
\hline $\begin{array}{l}\text { Hadjiyianni } \\
\quad[25]\end{array}$ & $\begin{array}{l}\text { Insulin, naive, initiators of basal } \\
\text { insulin }\end{array}$ & $\begin{array}{l}\text { Continuers vs interrupters } \\
\text { vs discontinuers: } 1 \text { year }\end{array}$ & $\begin{array}{l}\text { Adjusted costs (in yen) } \\
\text { Those who interrupted or discontinued had } \\
\text { higher costs of hospitalization than } \\
\text { continuers } \\
\text { Total costs did not differ among the } 3 \text { groups } \\
\text { Total medical costs did not differ among the } \\
3 \text { groups } \\
\text { Total pharmacy costs were higher for the } \\
\text { continuers }\end{array}$ \\
\hline $\begin{array}{l}\text { Chandran } \\
\text { [28] }\end{array}$ & $\begin{array}{l}\text { Insulin, naive, pen prescription } \\
\text { within study period }\end{array}$ & Adherence (MPR): 1 year & $\begin{array}{l}\text { Unadjusted costs } \\
\text { Average annual per-patient healthcare } \\
\text { expenditures }(P=0.007) \\
\text { Least adherent group (MPR }<0.20,11.0 \% \\
\text { of patients): } \$ 26,310 \\
\text { Most adherent group (MPR }>0.80 ; 34.6 \% \\
\text { of patients): } \$ 23,839 \\
\text { Average annual per-patient pharmacy } \\
\text { expenditures ( } P<0.001) \\
\text { Least adherent group: } \$ 5395 \\
\text { Most adherent group: } \$ 10,174\end{array}$ \\
\hline $\begin{array}{l}\text { Perez-Nieves } \\
\text { [9] }\end{array}$ & $\begin{array}{l}\text { Insulin, naïve, initiating basal } \\
\text { insulin }\end{array}$ & $\begin{array}{l}\text { Continuers vs interrupters } \\
\text { vs discontinuers } \\
\text { (identified } \\
\text { retrospectively) }\end{array}$ & $\begin{array}{l}\text { Cross-sectional survey } \\
\text { The three groups did not differ in reporting } \\
\text { the impact of insulin use on budget } \\
\text { management }\end{array}$ \\
\hline
\end{tabular}

a Text of article is unclear regarding whether the numbers reported are charges or costs of care GLP-1 RA glucose-like peptide-1 receptor agonist, $S D$ standard deviation, ED emergency department, $M P R$ medication possession ratio, $C I$ confidence interval

$(-0.45[-0.66,-0.24)] ; P<0.001]$ during the follow-up period. Such results typically represent the proportion of change in mean costs between the groups. Pharmacy costs were higher for the persistent group. 


\section{Insulin Studies}

See Table 4. Pharmacy costs, if measured, were invariably higher in the adherent or persistent groups, and medical costs (excluding pharmacy), if measured, were generally lower. As in the study by Buysman et al. [34], some insulin studies reported that the savings in medical costs were about equal to the pharmacy cost increase, and others, as in the study by Lin et al. [31], reported that persisters had a net savings in total costs.

\section{DISCUSSION}

The 34 studies obtained from this review reported on the relationships of adherence or persistence to the outcomes of glycemic control, healthcare resource use, and cost for adults with T2DM using injectable antihyperglycemics. Studies varied widely with respect to medications used, naïveté to index medications, study population characteristics, concurrence of the periods of measurement of adherence and the outcomes, study duration, definitions of adherence and outcome, and control by covariates. Most studies used conventional statistical methods and were not sufficiently sophisticated to account for the bidirectional and dynamic relationships between adherence and health outcomes $[47,48]$. For example, one such hypothetical relationship may be that "improvement in health outcomes over time may lead to better adherence and better adherence may lead to improvement in outcomes." Furthermore, other time-varying relevant covariates (e.g., glycemic control, frequency of healthcare visits, adherence to diet or exercise) may also dynamically interact with medication adherence. Advanced and more complex statistical models, e.g., marginal structural models [49-51], can account for these dynamic time-varying relationships, but such a model only appeared in 1 study in this review [15].

In spite of these limitations, most studies showed a benefit to adherence and persistence, confirming results from clinical trials. In general, persistence was associated with improved glycemic control. Patients who continued to take their medications demonstrated decreases from baseline HbA1c over the study periods. Patients who discontinued medications tended to revert back to study baseline HbA1c. Estimates of the effect of persistence on glycemic control appeared to be similar in the insulin and GLP-1 RA studies reviewed here, with 1-year HbA1c decreases approximating $1.0 \%$ for persisters.

Adherence studies using MPR or PDC measures from claims data often failed to make a distinction between persistence and adherence, generally categorizing the nonpersisters into the nonadherent group and making the interpretation of medication adherence more complex. This distinction was examined in the study by Buysmann et al. [34], where patients were independently classified as adherers and persisters, and where, at 1 year, a higher odds ratio (OR) was reported for reaching the HbA1c goal of $<7.0 \%$ for persisters vs nonpersisters $(\mathrm{OR}=2.34)$ compared with adherers vs nonadherers $(\mathrm{OR}=1.84)$. These studies also failed to report estimates of change in $\mathrm{HbA1c}$ per unit change in the adherence measure, except for Egede et al. [11], which reported an estimate of a $0.05 \%$ HbA1c reduction for each percentage increase in MPR. None of the GLP-1 RA studies reported such estimates.

However, studies that focused on adherence using patient-reported measures (all studies of insulin use) were able to describe the deviation from prescribed treatment that appeared associated with worse glycemic control $[10,12,13,22,24]$. For example, Linetsky et al. [22] showed that "no missed shots" was associated with a lower HbA1c (drop of $0.19 \%$ [0.05-0.34\%]) compared to all others who missed shots, and Aikens and Piette [24] found that each nonadherence unit in the Morisky score (range 0-4) was associated with a $0.16 \%$ increase in HbA1c at 6 months. Such studies, although elementary in design, begin to quantify how compliant patients must be to achieve the benefits of the medication.

Adherence and persistence were also associated with decreased healthcare resource use. Similarly, there was a fairly consistent relationship between adherence or persistence and 
decreased healthcare costs, although for some, the reduction in medical costs was about equal to the increase in pharmacy costs for adherent patients. These studies generally used a government or payer perspective, and did not consider the potential benefits that might accrue to patients, such as improved quality of life or fewer disability days.

Outcomes such as healthcare resource use and healthcare costs analyzed in cohort studies using administrative/claims data were often measured concurrently with adherence or persistence over the typical 1-year follow-up. Interpreting the results of such analyses as 'causal' is challenging, due to the temporal overlap of events. However, it may be that longer follow-up is required to demonstrate healthcare cost savings. In the second year of a 2-year study, Li et al. documented that medical costs for patients who were persistent with liraglutide were lower than the first year medical costs when compared to those patients using sitagliptin [52].

Furthermore, these studies could not directly measure the more subtle aspects of medication adherence. Compared to discontinuation, adherence is a more complex attribute of medication utilization, and it is harder to calculate, particularly with respect to injectables. Wastage due to inappropriate storage, transportation, or administration is problematic for injectables and cannot be measured from such administrative/claims data sources.

As noted above, adherence studies using claims data often failed to make a distinction between persistence and adherence. This imprecise measurement of adherence or persistence in claims-based studies regarding glycemic control also occurred in the resource use and cost studies. The rudimentary categorization of adherers and persisters makes these claims-based studies difficult to interpret. Fundamentally, these studies fail to distinguish between those who are nonadherent/nonpersistent based on doctors' orders (e.g., altering doses or stopping medications due to side effects) vs those who are nonadherent/nonpersistent based on personal decisions. Moreover, in situations where it is clear that the doctor has recommended medication (e.g., written prescriptions for it), they fail to distinguish between nonadherence/nonpersistence that is intentional (based on active decision-making) versus unintentional (e.g., due to forgetting, or due to the complexity of treatment) [53].

Ascher-Svanum et al. [8] found that the probability of restarting insulin therapy among early discontinuers was high $(90.3 \%)$, pointing not only to a low treatment attrition rate but also to the realization that early discontinuation appears to reflect a temporary interruption rather than a true and complete cessation. Importantly, an earlier study, which looked at persistence over a 10 -year period, found similar results: most patients (57.9\%) discontinued antihyperglycemic medication treatment at some point, but most discontinuers restarted, with only $8-10 \%$ of insulin users discontinuing and never restarting [54]. These issues may lead to bias towards the null as the nonadherent or nonpersistent group would actually include adherent or healthier intentionally nonadherent patients.

\section{Limitations}

The review was conducted using predefined eligibility criteria and conformed to PRISMA guidelines, and this contributes to the strength of the conclusions. However, limitations to this methodology include: (1) restriction to publications in the study period, and (2) the potential not to include articles that were lacking in the keywords used in the search but that did address the relationships of interest. Other limitations with respect to the generalizability of the data retrieved may have resulted from the various populations studied by the relevant articles (e.g., claims databases, national polls), discussion of both GLP-1 RA and insulin drug classes, and the methodological limitations outlined above. Another limitation is the potential misalignment between the quality score assigned to a study and the quality of the specific part of the results used for this review, particularly where the association of adherence with outcomes was a minor or secondary objective. 


\section{Looking Forward}

The results validate the effectiveness of GLP-1 RA and insulins for glycemic control, and suggest that there are consequences (both medical and financial) to nonadherence. The results can support steps to address reasons for nonadherence or discontinuation of these injectables.

Reasons for nonadherence have been classified by the World Health Organization [55] as "Five Dimensions of Adherence:" (1) health system: e.g., poor-quality provider-patient relationship, lack of access to healthcare, lack of continuity of care; (2) condition: e.g., asymptomatic chronic disease (lack of physical cues), mental health disorders (e.g., depression); (3) patient: e.g., physical impairments (e.g., vision problems, impaired dexterity), cognitive impairment, psychological/behavioral problems; (4) therapy: e.g., complexity of regimen, side effects; and (5) socioeconomic: e.g., low literacy, higher medication costs, poor social support.

This categorization suggests that there are multiple targets for adherence improvement strategies. Although much effort has been spent on the implementation of behavioral and technological strategies, these have largely been ineffective, as detailed in a recent Cochrane review [56]. Other avenues, including the use of health system incentives to encourage more contact and communication between healthcare providers and patients initiating treatment [57], reducing the complexity of medication regimens (including the use of alternative dosing strategies) $[44,58]$, and decreasing medication costs to the patient, may have the potential to help patients stay the course $[59,60]$.

To pave the way for the implementation of adherence improvement strategies, and to allow multiple strategies to be compared, future research will require the development of statistical models that identify the degree of adherence that would achieve a "minimal clinically important difference" (MCID) for glycemic control in adults with T2DM. An MCID defines a minimal threshold for clinically meaningful improvement; here, it would refer to the smallest change or difference in glycemic control considered meaningful, as determined, for example, by consensus within the clinical perspective $[61,62]$. Such consensus would rely on what is known about HbA1c improvement and clinical outcomes. Upon the determination of an MCID for glycemic control, a standard measure of patient adherence could be tested to identify the degree of adherence that achieves a HbA1c change of at least 1 MCID.

\section{CONCLUSIONS}

The results confirmed the effectiveness of injectable antihyperglycemic medications for glycemic control, and suggested that there are both medical and financial consequences to nonadherence. Over the last decade, there have been relatively few studies on the association of real-world adherence and persistence with health outcomes. Those identified generally used standard definitions and methods to compare medication adherence and persistence groups with the outcomes of glycemic control and healthcare resource use and costs. More sophisticated methodological approaches are necessary to account for the complex dynamics of both adherence and persistence over time, and make a better case for causal relationships to outcomes. Future research to establish a MCID for glycemic control in adult patients with T2DM, and to determine the required level of adherence to achieve that MCID, would assist in the determination of successful strategies for adherence improvement.

\section{ACKNOWLEDGEMENTS}

Funding. Funding for this study and for its publication was provided by Novo Nordisk, Inc., Plainsboro Township, NJ, USA. All authors had full access to all of the data in this study and take complete responsibility for the integrity of the data and the accuracy of the data analysis.

Medical Writing, Editorial, and Other Assistance. The authors acknowledge Dr. Lisa M. Korst, AMF Consulting, who was funded by 
Novo Nordisk, Inc. for manuscript preparation and editorial assistance, and Ms. Mariann E. Lucas for assistance with the literature search and with library services.

Authorship. All named authors meet the International Committee of Medical Journal Editors (ICMJE) criteria for authorship for this article, take responsibility for the integrity of the work as a a whole, and have given their approval for this version to be published.

Disclosures. Dr. Fridman is an employee of AMF Consulting, USA, and was contracted by Novo Nordisk, Inc. Dr. Hamersky is an employee of Novo Nordisk, Inc. Dr. Gamble is an employee of Novo Nordisk, Inc. Dr. Iyer is an employee of Novo Nordisk, Inc. Although employees of Novo Nordisk were involved in the design, collection, analysis, interpretation, and fact checking of information, the content of this manuscript, the interpretation of the data, and the decision to submit the manuscript for publication was made by the authors independently.

Compliance with Ethics Guidelines. This article is based on previously conducted studies and does not contain any studies with human participants or animals performed by any of the authors.

Data Availability. Data sharing is not applicable to this article as no original datasets were generated or analyzed during the current study.

Open Access. This article is distributed under the terms of the Creative Commons Attribution-NonCommercial 4.0 International License (http://creativecommons.org/licenses/ by-nc/4.0/), which permits any noncommercial use, distribution, and reproduction in any medium, provided you give appropriate credit to the original author(s) and the source, provide a link to the Creative Commons license, and indicate if changes were made.

\section{REFERENCES}

1. Asche C, LaFleur J, Conner C. A review of diabetes treatment adherence and the association with clincial and economic outcomes. Clin Ther. 2011;33(1):74-109.

2. Edelman SV, Polonsky WH. Type 2 diabetes in the real world: the elusive nature of glycemic control. Diabetes Care. 2017;40(11):1425-32.

3. Ottawa Hospital Research Institute, University of Oxford. Preferred Reporting Items for Systematic Reviews and Meta-Analyses (PRISMA) website. Available at: http://www.prisma-statement.org/ PRISMAStatement/PRISMAStatement.aspx. Accessed Apr 8, 2019.

4. Wells GA, Shea B, O'Connel D, et al. The Newcastle-Ottawa Scale (NOS) for assessing the quailty of nonrandomised studies in meta-analyses. Available at: http://www.ohri.ca/programs/clinical_ epidemiology/oxford.asp. Accessed Apr 8, 2019.

5. Wei W, Pan C, Xie L, Baser O. Real-world insulin treatment persistence among patients with type 2 diabetes. Endocr Pract. 2014;20(1):52-61.

6. Leiter LA, Boras D, Woo VC. Dosing irregularities and self-treated hypoglycemia in type 2 diabetes: results from the Canadian cohort of an international survey of patients and healthcare professionals. Can J Diabetes. 2014;38(1):38-44.

7. Wang L, Wei W, Miao R, Xie L, Baser O. Real-world outcomes of US employees with type 2 diabetes mellitus treated with insulin glargine or neutral protamine Hagedorn insulin: a comparative retrospective database study. BMJ Open. 2013;3:e002348.

8. Ascher-Svanum H, Lage MJ, Perez-Nieves M, et al. Early discontinuation and restart of insulin in the treatment of type 2 diabetes mellitus. Diabetes Ther. 2014;5(1):225-42.

9. Perez-Nieves M, Ivanova JI, Hadjiyianni I, et al. Basal insulin initiation use and experience among people with type 2 diabetes mellitus with different patterns of persistence: results from a multi-national survey. Curr Med Res Opin. 2017;33(10):1833-42.

10. Linetzky B, Jiang D, Funnell MM, Curtis BH, Polonsky WH. Exploring the role of the patientphysician relationship on insulin adherence and clinical outcomes in type 2 diabetes: insights from the MOSAIc study. J Diabetes. 2017;9(6):596-605.

11. Egede LE, Gebregziabher M, Echols C, Lynch CP. Longitudinal effects of medication nonadherence 
on glycemic control. Ann Pharmacother. 2014;48(5):562-70.

12. Osborn CY, Gonzalez JS. Measuring insulin adherence among adults with type 2 diabetes. J Behav Med. 2016;39(4):633-41.

13. DiBonaventura M, Wintfeld N, Huang J, Goren A. The association between nonadherence and glycated hemoglobin among type 2 diabetes patients using basal insulin analogs. Patient Prefer Adherence. 2014;8:873-82.

14. Anderten H, Dippel FW, Kostev K. Early discontinuation and related treatment costs after initiation of basal insulin in type 2 diabetes patients: a German primary care database analysis. J Diabetes Sci Technol. 2015;9(3):644-50.

15. Ayyagari R, Wei W, Cheng D, Pan C, Signorovitch J, $\mathrm{Wu}$ EQ. Effect of adherence and insulin delivery system on clinical and economic outcomes among patients with type 2 diabetes initiating insulin treatment. Value Health. 2015;18(2):198-205.

16. Donnelly LA, Morris AD, Evans JM. Adherence to insulin and its association with glycaemic control in patients with type 2 diabetes. QJM. 2007;100(6):345-50.

17. Wu EQ, Zhou S, Yu A, et al. Outcomes associated with insulin therapy disruption after hospital discharge among patients with type 2 diabetes mellitus who had used insulin before and during hospitalization. Endocr Pract. 2012;18(5):651-9.

18. Mashitani T, Hayashino $\mathrm{Y}$, Okamura $\mathrm{S}$, et al. Patient-reported adherence to insulin regimen is associated with glycemic control among Japanese patients with type 2 diabetes: Diabetes Distress and Care Registry in Tenri (DDCRT 3). Diabetes Res Clin Pract. 2013;100(2):189-94.

19. Sambamoorthi U, Garg R, Deb A, Fan T, Boss A. Persistence with rapid-acting insulin and its association with A1C level and severe hypoglycemia among elderly patients with type 2 diabetes. Curr Med Res Opin. 2017;33(7):1309-16.

20. Kindmalm L, Melander A, Nilsson JL. Refill adherence of antihyperglycaemic drugs related to glucose control (HbA1c) in patients with type 2 diabetes. Acta Diabetol. 2007;44(4):209-13.

21. Yavuz DG, Ozcan S, Deyneli O. Adherence to insulin treatment in insulin-naive type 2 diabetic patients initiated on different insulin regimens. Patient Prefer Adherence. 2015;9:1225-31.

22. Linetzky B, Curtis B, Frechtel G, et al. Challenges associated with insulin therapy progression among patients with type 2 diabetes: Latin American
MOSAIc study baseline data. Diabetol Metab Syndr. 2016;8:41.

23. Nishimura A, Harashima SI, Fukushige $H$, et al. A large difference in dose timing of basal insulin introduces risk of hypoglycemia and overweight: a cross-sectional study. Diabetes Ther. 2017;8(2):385-99.

24. Aikens JE, Piette JD. Longitudinal association between medication adherence and glycaemic control in type 2 diabetes. Diabet Med. 2013;30(3):338-44.

25. Hadjiyianni I, Desai U, Suzuki S, et al. Basal insulin persistence, associated factors, and outcomes after treatment initiation: a retrospective database study among people with type 2 diabetes mellitus in Japan. Diabetes Ther. 2017;8(1):149-66.

26. Cobden D, Lee WC, Balu S, Joshi AV, Pashos CL. Health outcomes and economic impact of therapy conversion to a biphasic insulin analog pen among privately insured patients with type 2 diabetes mellitus. Pharmacotherapy. 2007;27(7):948-62.

27. Kleinman NL, Schaneman JL, Lynch WD. The association of insulin medication possession ratio, use of insulin glargine, and health benefit costs in employees and spouses with type 2 diabetes. J Occup Environ Med. 2008;50(12):1386-93.

28. Chandran A, Bonafede MK, Nigam S, Saltiel-Berzin R, Hirsch LJ, Lahue BJ. Adherence to insulin pen therapy is associated with reduction in healthcare costs among patients with type 2 diabetes mellitus. Am Health Drug Benefits. 2015;8(3):148-58.

29. Perez-Nieves M, Kabul S, Desai U, et al. Basal insulin persistence, associated factors, and outcomes after treatment initiation among people with type 2 diabetes mellitus in the US. Curr Med Res Opin. 2016;32(4):669-80.

30. Kalirai S, Duan R, Liu D, Reed BL. Economic impact of treatment duration and persistence with basal insulin in previously insulin-naive users. J Manag Care Spec Pharm. 2017;23(3):327-36.

31. Lin J, Lingohr-Smith M, Fan T. Real-world medication persistence and outcomes associated with basal insulin and glucagon-like peptide 1 receptor agonist free-dose combination therapy in patients with type 2 diabetes in the US. Clinicoecon Outcomes Res. 2017;9:19-29.

32. Levin PA, Wei W, Zhou S, Xie L, Baser O. Outcomes and treatment patterns of adding a third agent to 2 OADs in patients with type 2 diabetes. J Manag Care Spec Pharm. 2014;20(5):501-12. 
33. Durden E, Lenhart G, Lopez-Gonzalez L, Hammer M, Langer J. Predictors of glycemic control and diabetes-related costs among type 2 diabetes patients initiating therapy with liraglutide in the United States. J Med Econ. 2016;19(4):403-13.

34. Buysman EK, Liu F, Hammer M, Langer J. Impact of medication adherence and persistence on clinical and economic outcomes in patients with type 2 diabetes treated with liraglutide: a retrospective cohort study. Adv Ther. 2015;32(4):341-55.

35. Carls GS, Tuttle E, Tan RD, et al. Understanding the gap between efficacy in randomized controlled trials and effectiveness in real-world use of GLP-1RA and DPP4 therapies in patients with type 2 diabetes. Diabetes Care. 2017;40(11):1469-78.

36. Buysschaert M, Preumont V, Oriot PR, et al. Oneyear metabolic outcomes in patients with type 2 diabetes treated with exenatide in routine practice. Diabetes Metab. 2010;36(5):381-8.

37. Varanasi A, Chaudhuri A, Dhindsa S, et al. Durability of effects of exenatide treatment on glycemic control, body weight, systolic blood pressure, C-reactive protein, and triglyceride concentrations. Endocr Pract. 2011;17(2):192-200.

38. Ivanyi T, Fovenyi J, Faludi P, et al. Long-term effects of adding exenatide to a regimen of metformin and/or sulfonylurea in type 2 diabetes: an uncontrolled, open-label trial in Hungary. Clin Ther. 2012;34(6):1301-13.

39. Chen YC, Lee CT, Lin BJ, Chang YY, Shi HY. Impact of pay-for-performance on mortality in diabetes patients in Taiwan: a population-based study. Medicine. 2016;95(27):e4197.

40. Polonsky WH, Fisher L, Hessler D, Edelman SV. Development of a new measure for assessing insulin delivery device satisfaction in patients with type 1 and type 2 diabetes. Diabetes Technol Ther. 2015;17(11):773-9.

41. Kokoszka A. Treatment adherence in patients with type 2 diabetes mellitus correlates with different coping styles, low perception of self-influence on disease, and depressive symptoms. Patient Prefer Adherence. 2017;11:587-95.

42. Carls GS, Tan R, Zhu JY, Tuttle E, Yee J, Edelman SV, et al. Real-world weight change among patients treated with glucagon-like peptide-1 receptor agonist, dipeptidyl peptidase-4 inhibitor and sulfonylureas for type 2 diabetes and the influence of medication adherence. Obes Sci Pract. 2017;3(3):342-51.

43. Slabaugh SL, Bouchard JR, Li Y, Baltz JC, Meah YA, Moretz DC. Characteristics relating to adherence and persistence to basal insulin regimens among elderly insulin-naïve patients with type 2 diabetes: pre-filled pens versus vials/syringes. Adv Ther. 2015;32(12):1206-21.

44. Stephenson JJ, Raval AD, Kern DM, Bae JP. Nonadherence to basal insulin among patients with type 2 diabetes in a US managed care population: results from a patient survey. Diabetes Obes Metab. 2018;20(11):2700-4.

45. Cramer JA, Roy A, Burrell A, et al. Medication compliance and persistence: terminology and definitions. Value Health. 2008;11(1):44-7.

46. Tuchscherer RM, Thompson AM, Trujillo JM. Semaglutide: the newest once-weekly GLP-1 RA for type 2 diabetes. Ann Pharmacother. 2018;52(12):1224-32.

47. DiMatteo MR, Giordani PJ, Lepper HS, Croghan TW. Patient adherence and medical treatment outcomes: a meta-analysis. Med Care. 2002;40(9):794-811.

48. Barnett A, Allsworth J, Jameson K, Mann R. A review of the effects of antihyperglycaemic agents on body weight: the potential of incretin targeted therapies. Curr Med Res Opin. 2007;23(7):1493-507.

49. Robins J, Hernan M, Babette B. Marginal structural models and causal inference in epidemiology. Epidemiol. 2000;11(5):550-60.

50. Hernan MA, Brumback B, Robins JM. Marginal structural models to estimate the causal effect of zidovudine on the survival of HIV-positive men. Epidemiol. 2000;11(5):561-70.

51. Robins JM. Marginal structural models versus structural nested models as tools for causal inference. In: Halloran E, Berry D, editors. Statistical models in epidemiology: the environment and clinical trials. New York: Springer; 1999.

52. Li Q, Ganguly R, Ganz ML, Gamble C, Dang-Tan T. Real-world clinical effectiveness and cost savings of liraglutide versus sitagliptin in treating type 2 diabetes for 1 and 2 years. Diabetes Ther. 2018;9:1279-93.

53. National Institute for Health and Care Excellence (NICE). Medicines adherence: involving patients in decisions about prescribed medicines and support in adherence. Clinical guideline 2009. Available at: https://www.nice.org.uk/guidance/cg76/chapter/ introduction. Accessed Apr 8, 2019.

54. Brown JB, Nichols GA, Glauber HS, Bakst A. Ten year follow-up of antidiabetic drug use, nonadherence, and mortality in a defined population with 
type 2 diabetes mellitus. Clin Ther. 1999;21(6):1045-57.

55. World Health Organization. Adherence to longterm therapy: evidence for action. 2003. Available at: http://www.who.int/chp/knowledge/ publications/adherence_report/en/. Accessed April 8, 2019.

56. Nieuwlaat R, Wilczynski N, Navarro T, et al. Interventions for enhancing medication adherence. Cochrane Database Syst Rev. 2014;(11)CD000011.

57. Ho PM, Bryson CL, Rumsfeld JS. Medication adherence: its importance in cardiovascular outcomes. Circulation. 2009;119(23):3028-35.

58. Marino AB, Cole SW, Nuzum DS. Alternative dosing strategies for liraglutide in patients with type 2 diabetes mellitus. Am J Health Syst Pharm. 2014;71(3):223-6.
59. Choudhry N, Avorn J, Glynn RJ, et al., for the PostMyocaridal Infarction Free Rx Event and Economic Evaluation (MI FREEE) Trial. Full coverage for preventive medications after myocardial infarction. New Engl J Med. 2011;365(22):2088-97.

60. Kaiser Family Foundation. Public opinion on prescription drugs and their prices. Slide presentation. Available at: https://www.kff.org/slideshow/publicopinion-on-prescription-drugs-and-their-prices/. Accessed Apr 8, 2019.

61. Jaeschke R, Singer J, Guyatt GH. Measurement of health status: ascertaining the minimal clinically important difference. Cont Clin Trials. 1989;10(4):407-15.

62. Wells G, Beaton D, Shea B, et al. Minimal clinically important differences: review of methods. J Rheumatol. 2001;28(2):406-12. 